\title{
Risk assessment in hypertrophic cardiomyopathy: contemporary guidelines hampered by insufficient evidence
}

\author{
Pascal P McKeown, ${ }^{1,2}$ Alison Rachel Muir ${ }^{2}$
}

\begin{abstract}
If it were not for the great variability among individuals, medicine might as well be a science and not an art. (Sir William Osler, 1892)
\end{abstract}

The sudden unexpected death of an individual at any age has a profound effect on the family and the wider community and this is particularly significant when it affects a young person. Hypertrophic cardiomyopathy remains one of the common causes of sudden cardiac death (SCD) in young individuals. ${ }^{1}$ In a cohort of 744 patients with hypertrophic cardiomyopathy from the USA and Italy, where 86 (12\%) died over an 8 (SD \pm 7$)$ year period of follow-up, three main modes of death were identified: sudden and unexpected (found mainly but not exclusively in adolescents and young adults); heart failure; and stroke, usually associated with the development of atrial fibrillation. ${ }^{2}$ The most common underlying pathophysiological mechanism leading to sudden death is the development of ventricular tachyarrhythmias occurring on a background of myocyte disarray, interstitial fibrosis, and arteriolar changes with contributory ischaemic and autonomic effects. ${ }^{1}$ Abnormalities of intercalated discs and gap junctions have also been reported. ${ }^{3}$ An integral component of clinical investigation and management of patients with hypertrophic cardiomyopathy is the assessment of risk of sudden death, as those individuals with a perceived significantly increased risk may be considered for prophylactic implantable cardioverter defibrillator (ICD) implantation.

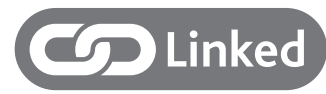

- http://dx.doi.org/10.1136/heartjnl-2012-303271

\footnotetext{
${ }^{1}$ School of Medicine, Dentistry and Biomedical Sciences, Queen's University Belfast, Belfast, Northern Ireland, UK; ${ }^{2}$ Department of Cardiology, Royal Victoria Hospital, Belfast Health and Social Care Trust, Belfast, Northern Ireland, UK

Correspondence to Prof Pascal P McKeown, Centre for Medical Education, School of Medicine, Dentistry and Biomedical Sciences, Queen's University Belfast, Whitla Medical Building, 97 Lisburn Road, Belfast, Northern Ireland BT9 7BL, UK; p.p.mckeown@qub.ac.uk
}

The estimated prevalence of hypertrophic cardiomyopathy is 1 in 500 but many individuals have a subclinical phenotype. Many patients with this condition have an excellent prognosis similar to that of the background population and these individuals are characterised by the absence of major risk factors for sudden death. ${ }^{4}$ Several risk factors for sudden death have been identified. ${ }^{15}$ The generally accepted major risk factors include: family history of sudden death; unexplained syncope; non-sustained ventricular tachycardia (NSVT) on ambulatory monitoring; severe hypertrophy $(\geq 30 \mathrm{~mm})$; and abnormal blood pressure response to exercise (although this risk may be relevant only in those under the age of 40 years). ${ }^{67}$ Additional 'risk modifiers' have also been reported and include the presence of significant left ventricular outflow tract obstruction (LVOTO), significant late gadolinium enhancement (LGE) on cardiac MRI scanning and 'high risk' genotype, as well as other less commonly seen complications, such as exercise-induced ventricular tachyarrhythmias, the presence of a left ventricular aneurysm and late systolic dysfunction. ${ }^{15}$

There are difficulties, however, in the interpretation of these risk factors in the context of an individual patient with hypertrophic cardiomyopathy. Of note, many publications relating to risk assessment in hypertrophic cardiomyopathy have come from a limited number of centres where there may have been both a referral and ascertainment bias-patients attending these regional centres are likely to have had a more severe expression of phenotype, leading to early reports suggesting that there was a high risk (up to $6 \%$ per year) of sudden death. ${ }^{1}$ Some risk factors have shown statistical significance on univariate but not multivariate analysis, leading to the ACCF/AHA guidelines 'downgrading' NSVT and BP response to exercise as only being of significance if observed in the context of other major risk factors. ${ }^{7}$

Various authors have also used different definitions of individual risk factors. As a simple illustration, how does one define a family history of sudden death? In the papers which were quoted in a recent meta-analysis, ${ }^{8}$ a family history of premature SCD was defined in several different ways, including SCD in a first degree relative, SCD in $\geq 2$ relatives $<40$ years, SCD in $\geq 2$ first degree relatives $<40$ years, and SCD in $\geq 1$ relatives with hypertrophic cardiomyopathy or SCD in $\geq 1$ close relatives without the diagnosis of hypertrophic cardiomyopathy $<50$ years, leaving the clinician in some difficulty when interpreting whether or not a family history is indeed significant. The 2011 ACCF/AHA guidelines $^{7}$ make a class IIa recommendation that it is reasonable to recommend an ICD for hypertrophic cardiomyopathy patients with a family history of SCD presumed due to hypertrophic cardiomyopathy in one or more first-degree relatives; however, this implies that, if an individual in a family dies due to hypertrophic cardiomyopathy, all surviving affected family members may be offered an ICD irrespective of their phenotype, an approach which seems rather alarmist.

The newer 'risk modifiers' also pose clinical dilemmas. Some studies have shown higher rates of SCD among patients with resting LVOTO, with reduction in risk following relief of obstruction via surgical myectomy. ${ }^{9}$ Fibrosis on histological examination is a common finding in hypertrophic cardiomyopathy and it was initially reported that the presence of LGE on MRI scanning was a risk factor for SCD. However, LGE has been reported in over $60 \%$ of patients with hypertrophic cardiomyopathy, is particularly common in those with extensive hypertrophy, and does appear to be associated with an increased risk of ventricular arrhythmias but is not consistently independently associated with an increased risk for SCD. ${ }^{10} 11$ To date, studies have been underpowered and have shown significant heterogeneity. ${ }^{12} 13$

Some early reports had suggested that there may be a link between genotype and sudden death, in particular with variants in the troponin $\mathrm{T}$ gene, ${ }^{14}$ but more recently, patients with troponin $\mathrm{T}$ mutations were found to have rates of SCD similar to those reported in large referral populations. ${ }^{15}$ On the other hand, some patients who are genotype positive but do not express any hypertrophy, also carry risk factors for SCD. ${ }^{16}$ It is increasingly recognised that $5-10 \%$ of patients with hypertrophic cardiomyopathy have more than one pathogenic variant (with compound or double heterozygosity associated with a more severe expression of phenotype), with some studies suggesting an increased rate of SCD. ${ }^{17}$ 
It is clear that standard programmed ventricular stimulation is not a useful investigation in risk assessment. ${ }^{1}$ More complex electrophysiological assessment, using techniques such as electrogram fractionation, may offer additional prognostic information, but the technique is invasive and there are limited datasets available. ${ }^{18}$

The main therapeutic option for reduction of sudden death is ICD implantation, but complications from these devices, including acute complications relating to the implant procedure, inappropriate shocks, lead failure, and long-term psychological effects are increasingly recognised. There is also some difficulty in determining whether or not an ICD discharge was lifesaving, as it is possible that some arrhythmias terminated by an ICD shock may have terminated spontaneously. The Heart Rhythm UK group has suggested that patients should have an SCD risk of $\geq 1 \%$ per year before considering ICD implantation. ${ }^{19}$ There is also the issue of the unpredictable timing of when a patient may require an ICD shock-in a modest number of patients there may be many years between ICD implant and first discharge. Decisions about implantation in young people are complex as they have most to lose in the setting of SCD, yet they wish to lead active lives but encounter devices with limited battery life and lead problems, leading to multiple invasive procedures over a lifetime. Schinkel et al have undertaken a meta-analysis and reported an overall low cardiac mortality after ICD implantation. ${ }^{20}$ While the appropriate intervention rate was $\sim 3.3 \%$, inappropriate interventions and complications were not uncommon $(4.8 \%$ and $3.4 \%$ per year, respectively). Newer devices, which do not require intravascular access, may be useful but there are only a small number of published studies. ${ }^{21}$

In the study by O'Mahony et al, ${ }^{22}$ the dataset from a cohort of 1606 patients with hypertrophic cardiomyopathy evaluated at their centre was analysed using the guideline documents issued in 2003 and 2011. ${ }^{7}$ During the study period (median 6.6 years, IQR 4.1-10.0), 302 patients received an ICD. The primary endpoint of SCD was defined as one of the following: witnessed or aborted SCD with or without documented ventricular fibrillation; death within $1 \mathrm{~h}$ of symptoms; nocturnal death with no antecedent history of worsening symptoms; or an appropriate ICD shock for ventricular tachycardia or fibrillation. They identified that the risk of SCD increased with multiple risk factors but not with a single risk factor. The area under the receiver operating characteristic curves at 1 and 5 years based on the two main algorithms ranged from 0.61 to 0.64 and, as such, had limited power to distinguish high risk from low risk individuals. As has been previously noted, the positive predictive accuracy of these risk algorithms is low, whereas the negative predictive value is good. It is important to note that the majority of events still occur in those perceived to be at low risk. This study, therefore, provides some evidence to suggest that the 2011 guidelines do not really help to distinguish high from low risk candidates.

It is important to remember that currently available algorithms classify risk factors as dichotomous variables, and these authors comment: 'it is implausible that a maximal wall thickness of $29 \mathrm{~mm}$ confers a significantly different risk than a value of $30 \mathrm{~mm} .^{22}$ They recognise that risk factors are not static and may change with time but they do not address the issue of 'gathering risk'. In this paper there was a single assessment of risk at baseline but it is important to remember that, over time, individuals may acquire additional risk factors with changes in symptoms, family history or follow-up investigations. Current guidelines recommend assessment at initial evaluation and on a period follow-up basis of 12-24 months. ${ }^{7}$ However, if two risk factors are 'gathered' at separate visits, the impact of this is unclear. In addition, it is important to note that many of the published datasets on risk factors are based on 'relative' rather than an accurate 'absolute' risk calculation.

It is also striking to note that the evidence base cited in the 2011 consensus document is mainly at level $\mathrm{B}$ or $\mathrm{C}^{7}$ There is a clear need for more evidence and longer term follow up to assess the usefulness of these algorithms. In view of the wide phenotypic expression of hypertrophic cardiomyopathy, future studies should include information from cohorts of patients, which are representative of hypertrophic cardiomyopathy patients in the general population. ${ }^{1}$ This will require international collaboration as well as the use of high quality registry data, an issue which has been recognised recently by the European Society of Cardiology. ${ }^{23}$ Ultimately, it requires expert input from the cardiology team and clear communication with an informed patient to ensure that both the art and science of medicine are utilised in the best interests of the patient.

Contributors Both authors contributed to the preparation of the article. PMcK takes responsibility for the overall content and acts as guarantor.

Competing interests None.

Provenance and peer review Commissioned;

internally peer reviewed.

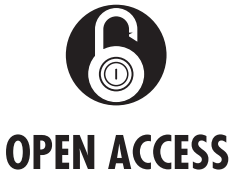

Open Access This is an Open Access article distributed in accordance with the Creative Commons Attribution Non Commercial (CC BY-NC 3.0) license, which permits others to distribute, remix, adapt, build upon this work non-commercially, and license their derivative works on different terms, provided the original work is properly cited and the use is noncommercial. See: http://creativecommons.org/licenses/ by-nc/3.0/

To cite McKeown PP, Muir AR. Heart 2013;99:511-513.

Published Online First 2 February 2013

Heart 2013:99:511-513.

doi:10.1136/heartjnl-2012-303363

\section{REFERENCES}

1 Maron BJ. Contemporary insights and strategies for risk stratification and prevention of sudden death in hypertrophic cardiomyopathy. Circulation 2010;121:445-56.

2 Maron BJ, Olivotto I, Spirito P, et al. Epidemiology of hypertrophic cardiomyopathy-related death: revisited in a large non-referral-based patient population. Circulation 2000;102:858-64.

3 Sepp R, Severs NJ, Gourdie RG. Altered patterns of cardiac intercellular junction distribution in hypertrophic cardiomyopathy. Heart 1996:76:412-17.

4 Kofflard MJ, Ten Cate FJ, van der Lee C, et al. Hypertrophic cardiomyopathy in a large community-based population: clinical outcome and identification of risk factors for sudden cardiac death and clinical deterioration. J Am Coll Cardiol 2003:41:987-93.

5 Gimeno JR, Tomé-Esteban M, Lofiego C, et al. Exercise-induced ventricular arrhythmias and risk of sudden cardiac death in patients with hypertrophic cardiomyopathy. Eur Heart J 2009;30:2599-605.

6 Maron BJ, McKenna WJ, Danielson GK, et al. American College of Cardiology/European Society of Cardiology Clinical Expert Consensus Document on Hypertrophic Cardiomyopathy: a report of the American College of Cardiology Foundation Task Force on Clinical expert Consensus Documents and the European Society of cardiology Committee for Practice Guidelines. Eur Heart J 2003:24:1965-91.

7 Gersh BJ, Maron BJ, Bonow RO, et al. ACCF/AHA guideline for the diagnosis and treatment of hypertrophic cardiomyopathy: executive summary: a report of the American College of Cardiology Foundation/American Heart Association Task Force on Practice Guidelines. Circulation 2011;124:2761-96.

8 Christiaans I, van Engelen K, van Langen IM, et al Risk stratification for sudden cardiac death in hypertrophic cardiomyopathy: systematic review of clinical risk markers. Europace 2010;12:313-21.

9 Dearani JA, Ommen SR, Gersh BJ, et al. Surgery insight: septal myectomy for obstructive hypertrophic cardiomyopathy - the Mayo Clinic experience. Nat Clin Pract Cardiovasc Med 2007:4:503-12.

10 O'Hanlon R, Grasso A, Roughton M, et al. Prognostic significance of myocardial fibrosis in hypertrophic cardiomyopathy. J Am Coll Cardiol 2010;56:867-74.

11 Bruder O, Wagner A, Jensen CJ, et al. Myocardial scar visualized by cardiovascular magnetic resonance imaging predicts major adverse events in patients 
with hypertrophic cardiomyopathy. I Am Coll Cardiol 2010;56:875-87.

12 Green JJ, Berger JS, Kramer CM, et al. Prognostic value of late gadolinium in clinical outcomes for hypertrophic cardiomyopathy. I Am Coll Cardiol Img 2012;5:370-7.

13 Ismail TF, Prasad SK, Pennell DJ. Prognostic importance of late gadolinium enhancement cardiovascular magnetic resonance in cardiomyopathy. Heart 2012;98:438-42.

14 Watkins H, McKenna WJ, Thierfelder $L$, et al. Mutations in the genes for cardiac troponin T and alpha-tropomyosin in hypertrophic cardiomyopathy. N Engl J Med 1995;332:1058-64.

15 Pasquale F, Syrris P, Kaski JP, et al. Long-term outcomes in hypertrophic cardiomyopathy caused by mutations in the cardiac troponin T gene. Circ Cardiovasc Genet 2012;5:10-17.

16 Michels M, Soliman O, Phefferkorn J, et al. Disease penetrance and risk stratification for sudden cardiac death in asymptomatic hypertrophic cardiomyopathy mutation carriers. Eur Heart $\mathrm{J}$ 2009;30:2593-8

17 Ingles J, Doolan A, Chiu C, et al. Compound and double mutations in patients with hypertrophic cardiomyopathy: implications for genetic testing and counselling. J Med Genet 2005; 42:e59.

18 Saumarez RC, Pytkowski M, Sterlinski M, et al. Paced ventricular electrogram fractionation predicts sudden cardiac death in hypertrophic cardiomyopathy. Eur Heart J 2008:29:1653-61.

19 Garratt CJ, Elliott P, Behr E, et al.; Heart Rhythm UK Familial Sudden Cardiac Death Syndromes Statement Development Group. Heart Rhythm UK position statement on clinical indications for implantable cardioverter defibrillators in adult patients with familial sudden cardiac death syndromes. Europace 2010; 12:1156-75.
20 Schinkel AF, Vriesendorp PA, Sijbrands EJ, et al. Outcome and complications after implantable cardioverter defibrillator therapy in hypertrophic cardiomyopathy: systematic review and meta-analysis. Circ Heart Fail 2012:5:552-9.

21 Cappato R, Smith WM, Hood MA, et al. Subcutaneous chronic implantable defibrillation systems in humans. I Interv Card Electrophysiol 2012;34: 325-32.

22 O'Mahony C, Tome-Esteban M, Lambiase P, et al. A validation study of the 2003 American College of Cardiology/European Society of Cardiology and 2011 American College of Cardiology Foundation/ American Heart Association risk stratification and treatment algorithms for sudden cardiac death in patients with hypertrophic cardiomyopathy. Heart 2013(99):534-41.

23 http://www.escardio.org/guidelines-surveys/eorp/ surveys/Pages/welcome.aspx (Accessed Jan 2 2013). 